\title{
DISCUSSION
}

\section{Interpretation by numerical modelling of changes of fracture system hydraulic conductivity induced by fluid injection}

\author{
T. R. HARPER and N. C. LAST (1989). Géotechnique, 39, No. 1, 1-11
}

\section{E. Vargas, Catholic University, Rio de Janeiro}

This discussion is focused on the flow part of Harper and Last's computer implementation. More specifically, I would like to address a point related to the use of expressions (9) and (7) in order to calculate pressure variations on nodal intersections of flow domains. These expressions are slightly modified and renumbered below

$$
\begin{aligned}
\Delta p & =((Q / V)+\dot{V}) C_{\mathrm{s}} \Delta t \\
q & =K_{\mathrm{f}} a(P / L) \\
K_{\mathrm{f}} & =C a^{2}
\end{aligned}
$$

where $a$ is the fracture opening.

According to the Authors, the values of $Q$ to be used in (10) are calculated by way of (11) assuming a linear pressure drop between flow domains $(P / L)$.

Bray (1981) (in Vargas, 1982) has shown that for a fracture of uniform aperture subjected to a rate of change of aperture $\mathrm{d} e / \mathrm{d} t$, the velocity and the head drop at a distance $l$ along its length are given by

$$
\begin{aligned}
q & =q_{0}-(1 / a)(\mathrm{d} a / \mathrm{d} t) l \\
\Delta h & =\left(1 / K_{\mathrm{f}}\right)\left(q_{0 l}-(1 / a)(\mathrm{d} a / \mathrm{d} t)\left(l^{2} / 2\right)\right)
\end{aligned}
$$

where $q_{0}$ is initial velocity.

Expressions (12) show that both the velocity and head along the fracture are affected by the rate $(\mathrm{d} a / \mathrm{d} t)$, the former linearly and the latter quadratically. It appears therefore that expression (11) should rigorously take into account the effect of $(\mathrm{d} a / \mathrm{d} t)$ in $q$. I have implemented a fracture flow-rigid and deformable blocks coupling similar to the Authors' (Vargas, 1982; Vargas, 1985) where flow along fractures was modelled by means of 1D finite elements taking into account the compressibility of the water, changes in fracture volume and linear or quadratic head variation along elements as suggested by (12).

The relevance of how one models the head variation between flow domains is illustrated by an example, depicted in Fig. 11(a). It shows two rigid blocks sitting on top of a third rectangular fixed block. Flow is considered to take place along fractures 1 and 2 only. Initially, the two upper blocks are left to 'consolidate' under their own weight $W_{\mathrm{b}}$ which is deliberately exaggerated in order to magnify the effects. Fig. 11(b) shows the loads imposed at each of the two upper blocks. The water force $P_{\mathrm{w}}$ corresponds to a constant pressure head of $40 \mathrm{~m}$ and a total head of $60 \mathrm{~m}$ since the elevation head is $20 \mathrm{~m}$ for both elements. In the second stage, the total heads at nodes 1 and 3 are reduced from 60 to $40 \mathrm{~m}$. As the upward force is reduced, the blocks move down to produce a change in the fracture volume, causing an increase in flow and in the total head at mode 2. Fractures 1 and 2 have the following characteristics: contact normal stiffness $10^{10} \mathrm{~N} / \mathrm{m}$, contact shear stiffness $10^{10} \mathrm{~N} / \mathrm{m}$ and $C=6 \times 10^{5}(\mathrm{~ms})^{-1}$ (to be used in equation (12)). The objective of the exercise was to follow the variation of head at the central node with time. Bray (1981) obtained a semi-analytical solution for this problem which served to validate the numerical code developed by Vargas (1982).

Figures 12 and 13 show the results obtained considering two different initial apertures. In Fig. 13 oscillations that occurred at the first few cycles of calculation for the quadratic head variation case are not shown, for the sake of clarity. It appears that the rate of head variation is greater when considering a linear head variation along elements is considered and this difference is more pronounced as the initial aperture decreases. The long-term head at node 2 is obviously $40 \mathrm{~m}$ and it should not be affected by the transient phase. Although the illustrative example may not be representative of all conditions, it seems that for time dependent problems it may be relevant how one models the variation in head between flow domains.

It is not clear, however, what effect it might have on quasi-static problems (such as those analysed by the Authors) where non-linearities such as slip, separations and block rolations may be present.

\section{Authors' reply}

The discussion by E. Vargas concentrates on the numerical formulation of the block model as used by Harper and Last, and in particular on the fluid flow equations and their implementation.

Vargas has implemented a model which includes 'linear or quadratic head variation along elements as suggested by equation (12).' Details of 


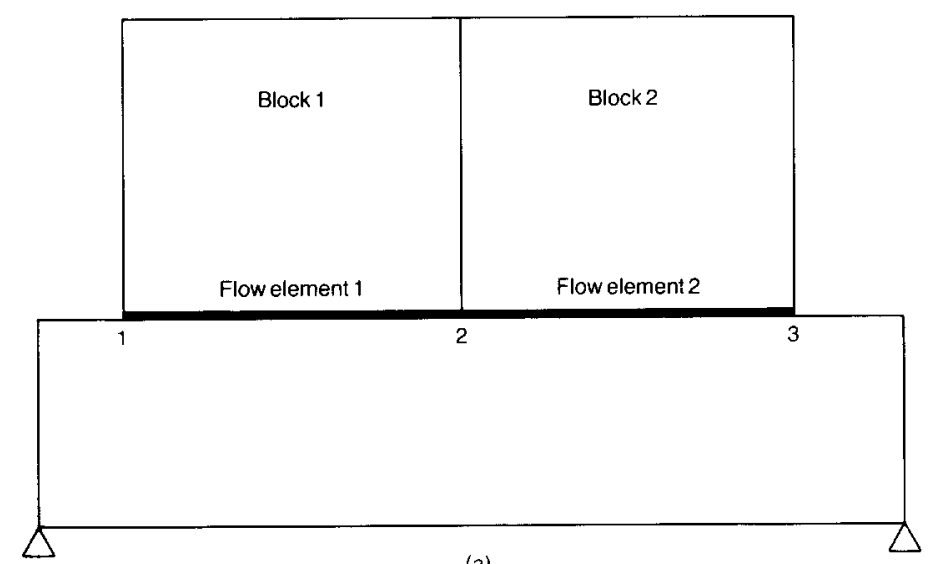

(a)

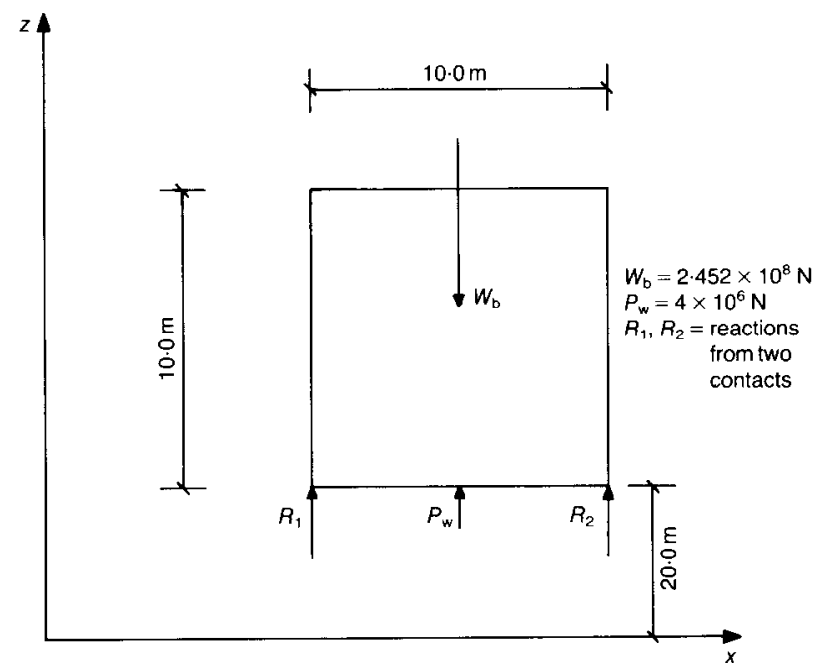

(b)

Fig. 11. Three blocks coupled problem

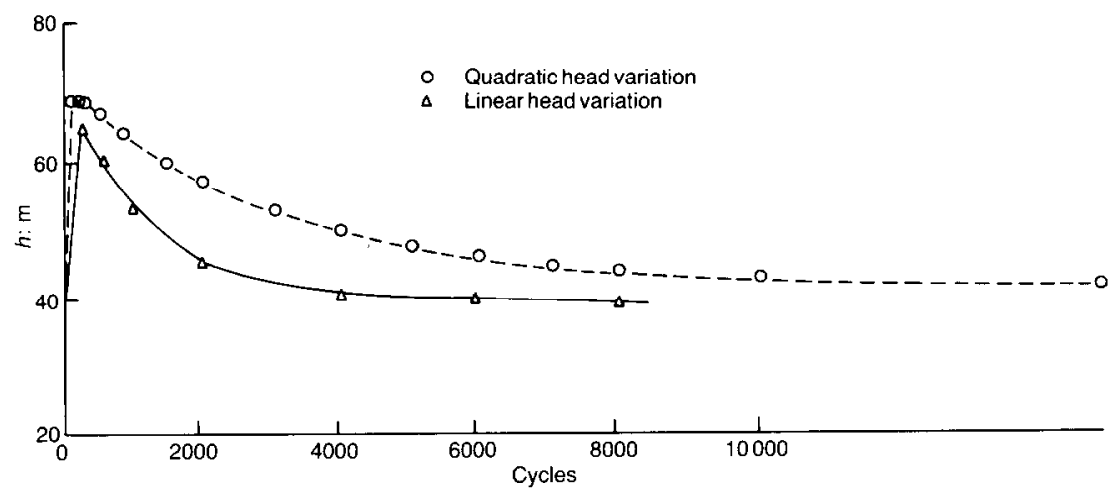

Fig. 12. Head at node $2 x$ iteration cycles: initial aperture $=10^{-3} \mathrm{M}$; each calculation cycle $=10^{-4} \mathrm{~s}$ 


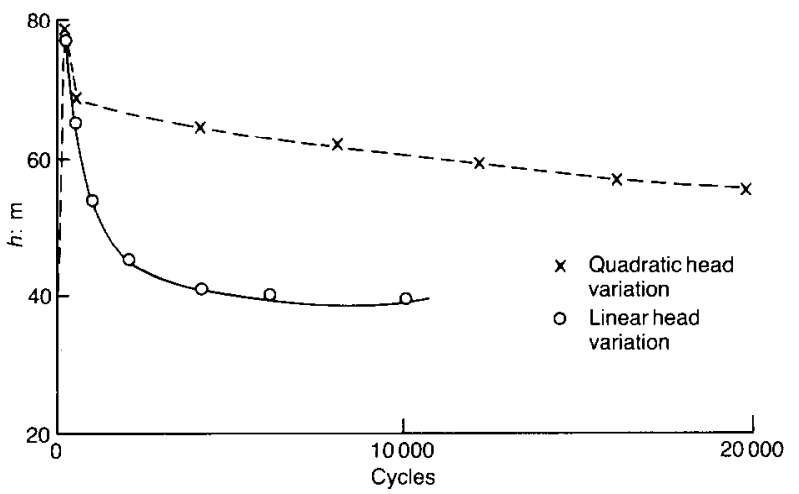

Fig. 13. Head at node $2 x$ iteration cycles: initial aperture $=5 \times 10^{-4} \mathrm{M}$; each calculation cycle $=10^{-4} \mathrm{~s}$

the formulation (e.g. how compressibility is taken into account) are not provided. Equations (12) indicate that the velocity and head are linearly dependent on the rate of closure, but are respectively linearly and quadratically dependent on the distance along the joints. Presumably, therefore, in the 'linear' case the second term in the second of equations (12) is ignored. If so, then the pressure change associated with the change in joint volume is apparently omitted. In contrast, the Authors' linear UDEC model does account for the effects of $\mathrm{d} a / \mathrm{d} t$, through the second term of equation (10)

$$
\begin{aligned}
\dot{v} & =\left(\mathrm{d} a^{*} / \mathrm{d} t\right) \cdot(L / V) \\
& =(1 / a) \cdot(\mathrm{d} a / \mathrm{d} t)
\end{aligned}
$$

for a joint element of constant length $L$. The first term in the equation $Q$, is the net flow into the fluid element. The advantage of casting the equation in the original form, equation (10), is that it is general and can be applied to any fluid filled domain in the system (i.e. voids as well as fractures). Furthermore, the effects of fluid loss into the blocks can be easily included. I have used our model to examine the problem presented in the discussion. However, I do not have full details of the input parameters or of the boundary conditions applied to the blocks. For example, can the top blocks rotate? They should, if not specifically constrained, but if they do, the assumption of a uniform rate of fracture closure is violated. For the purposes of experimentation, I used the material parameters from our paper, and assumed that the top blocks could only translate vertically. I can then reproduce the characteristic behaviour reported in the discussion. In fact, the response is qualitatively much closer to the 'quadratic' than to the 'linear' simulations. If the rate of fracture closure term is omitted, not surprisingly the pressure decline is much more rapid. However, a strict comparison would only be meaningful if the similarity of the boundary value problem is confirmed.

Thus, the main question appears to centre on whether or not the rate of fracture closure and its effects have been implemented in a rigorous manner. To date, I have been unable to obtain the references cited by Vargas: these may clarify the interpretation of equations (12). In particular, they may resolve the following difficulty I have with the use of the term 'velocity' to describe the effects of the joint deformations on the flow regime. Perhaps a better way to describe the (1/ $a \cdot \mathrm{d} a / \mathrm{d} t \cdot l)$ term of equation (12) is the rate of change of volume per unit cross-sectional area. Consider the case in which a fluid-filled joint is sealed at both ends and is subjected to a uniform change in aperture. The volume of the joint changes and the pressure in the joint changes uniformly, but there is no flow along the length of the joint, and hence the 'velocity' is zero. A similar argument can be applied for the case of uniform leak-off into the rock matrix. These effects are correctly incorporated into the UDEC model through equations (10) and (11).

Assuming that the questions raised above result mainly from a lack of detailed information, and that Vargas's model represents a complete higher-order implementation of the flow equations (with a quadratic spatial variation of head), then, as with other numerical formulations, it is likely that in most problems the true response will be more closely simulated, as illustrated by the example in the discussion. However, in coupled problems, there seems little justification in adopting a higher-order representation of one field variable compared to another. For example, if there is a quadratic variation of joint fluid pressures in the block model, is there also a quadratic variation of joint stresses, block stresses and block fluid pressures? In our model, we have been broadly consistent in this respect. There is insufficient detail in the discussion to ascertain whether 
or not this is the case in Vargas's model. It is clear, however, that a uniform rate of fracture closure is assumed. This appears to be inconsistent with a higher-order variation of fluid pressure or rock stress. If the model does correctly include a higher-order representation of the primary field variables, this undoubtedly implies a much more complex code, albeit potentially more accurate.

In conclusion, our overall aim was to investigate by numerical modelling the effect of fluid injection on a jointed, saturated, deformable rock mass, where the interaction between the fluid and rock is important. In particular, we sought to gain a better understanding of the characteristic behaviour resulting from injection as a function of two key variables, fracture pattern and in situ stress state. The model is a simplified and idealized representation of reality. Nevertheless, we feel that it encapsulates the key features, including the highly non-linear effects resulting from slip, dilation and stress-level dependent hydraulic conductivity. While accepting that in many instances higher order elements can provide greater accu- racy, we feel that we achieved our overall aim. It is good that Vargas has brought his formulation to my attention. I am certain that there are classes of problems for which use of the higherorder elements will be justified. This work warrants further discussion.

\section{ACNOWLEDGEMENT}

The author is grateful to Dr M. I. Asgian for the useful discussions held during the formulation of this reply.

\section{REFERENCES}

Bray, J. W. (1981). Personal communication. Rock Mechanics Section, Imperial College London.

Vargas, E. (1982). Development and application of numerical models to simulate the behaviour of fractured rock masses. $\mathrm{PhD}$ thesis, University of London.

Vargas, E. (1985). Application of the dynamic relaxation technique to coupled stress-flow problems in fractured media. Proc. Int. Sth Int. Conf. Num. Methods in Geomechanics Nagoya, pp. 681-688. 